\title{
Propuesta de innovación docente en Derecho Procesal: jurisdicción y competencia a través de un taller de fomento de las relaciones saludables y prevención de la violencia de género
}

\section{A proposal for teaching innovation in Procedural Law: jurisdiction and judicial competence through a workshop in order to promote healthy relationships and prevent gender violence}

María LUISA Domínguez BarRagán

Universidad de Sevilla

Departamento de Derecho Procesal

mdominguez16@us.es

ORCID: https://orcid.org/0000-0003-4796-3624

DOI: http://dx.doi.org/10.12795/9788447231003.016

Pp.: $333-350$ 


\section{Breve descripción del contexto}

Sin duda, el año 2020 es un año muy complicado en todos los sentidos. Esa dificultad ha sido trasladada a las aulas universitarias, donde el curso 2020/2021 ha empezado de forma radicalmente distinta a la que todos estábamos acostumbrados. El alumno ha llegado algo desorientado pero también muy expectante, a la espera de cómo va a poder desarrollarse un curso que, desde el inicio, está lleno de incertidumbres. El hecho de que un mismo grupo esté subdividido en pequeños grupos a través del sistema bimodal no fomenta la integración ni la participación en el aula. En este clima cualquier experiencia novedosa hace que el alumnado de primeras se muestre reticente, pero rápidamente olvide toda esa angustiosa nueva forma de asistir a clase y esté más receptivo. A estas ya difíciles circunstancias se le suma que el grupo donde se ha aplicado el CIMA es un grupo de segundo curso del Grado en Derecho que, en consecuencia, ha tenido un primer curso de Grado en Derecho absolutamente anormal debido al confinamiento. Además, durante la aplicación del CIMA se ha pasado del sistema bimodal al sistema online.

La asignatura que imparto es, como ya se ha dicho, una asignatura cuatrimestral de segundo curso del Grado en Derecho, denominada Derecho Procesal I (aunque antes recibía el nombre de Introducción al Derecho Procesal, denominación por la que todavía es conocida). El grupo es numeroso, pues hay 72 alumnos matriculados. Además, la dificultad que encierra la asignatura es alta, pues el derecho procesal es una materia árida que atiende a la manifestación práctica de las materias de contenido sustantivo, a lo que se suma la utilización de un lenguaje muy técnico. Es una disciplina de carácter formal, a la que estos alumnos se acercan por primera vez. Si bien es desconocida para ellos, es necesario que tengan unos mínimos conocimientos previos de otras materias (por ejemplo de derecho constitucional). Este es uno de los principales hándicaps de la materia, pues el alumno va a estudiar la organización y funcionamiento de los tribunales en general, teniendo

Ciclos de Mejora en el Aula (2020). Experiencias de Innovación Docente de la US Esta obra se distribuye con la licencia Creative Commons 
en cuenta que aún no ha cursado ni derecho administrativo, ni derecho mercantil ni tampoco derecho del trabajo, y de las materias civil y penal posee conocimientos limitados. Por ello, se hace imprescindible un acercamiento a la materia desde perspectivas conocidas para ellos, principalmente a través de la información proporcionada por los medios de comunicación. A todo esto, ha de sumarse la importancia de fomentar un sentido crítico para el futuro jurista.

Así, en el presente trabajo se presenta el diseño, desarrollo y evaluación de un CIMA centrado en uno de los tres pilares que conforman, de manera general, la disciplina, y que son: jurisdicción, acción y proceso. Concretamente, se aborda la explicación de la jurisdicción (parte II), lección tercera del Manual del Profesor Martín Ostos (Martín Ostos, 2020) -lecciones tercera y cuarta del proyecto docente-, dentro de la parte dedicada a los conceptos fundamentales de la asignatura. Son conceptos importantes que luego se ampliarán en los cursos siguientes y que son la base para estructurar el sistema de justicia y, por ende, el conjunto del ordenamiento jurídico. El jurista ha de tener unos buenos cimientos procesales, ya que éstos le facilitarán en alto grado su trabajo.

\section{Diseño previo del Ciclo de Mejora en el Aula}

\section{Mapa de contenidos}

Al tratarse de la segunda parte de la explicación sobre el pilar de la jurisdicción, ya la pregunta de base para la arquitectura del conocimiento no puede ser (como sería lo normal) el concepto de jurisdicción, sino que se ha de ir más allá. El planteamiento se centra más que en el propio concepto en su aplicación práctica (no obstante, es imprescindible el manejo del término). 
Lo que busca el mapa de contenidos es la unión de todos los conceptos de forma que cobren sentido conjuntamente y no se estudien de manera individual, dando respuesta a dos cuestiones principales. Los alumnos tienen que saber diferenciar los distintos órdenes jurisdiccionales y las maneras de solventar los conflictos que pueden surgir con otras jurisdicciones, de unos órdenes con otros y dentro de los órganos de los propios órdenes. Dentro ya de cada orden, como manifestación de la propia jurisdicción, han de conocer los tres tipos de competencia (objetiva, territorial y funcional) con los conflictos que pueden generarse y dentro de cada órgano la forma práctica de atribución de los asuntos, es decir, el reparto. Como el conocimiento de los distintos órdenes conlleva, necesariamente, conocer la pirámide judicial en sus distintas versiones, se ha aprovechado para integrarlas dentro de este tema, aunque se estudien con una mayor profundidad en lecciones más avanzadas del temario.

Por ello, utilizamos el siguiente mapa de contenidos:

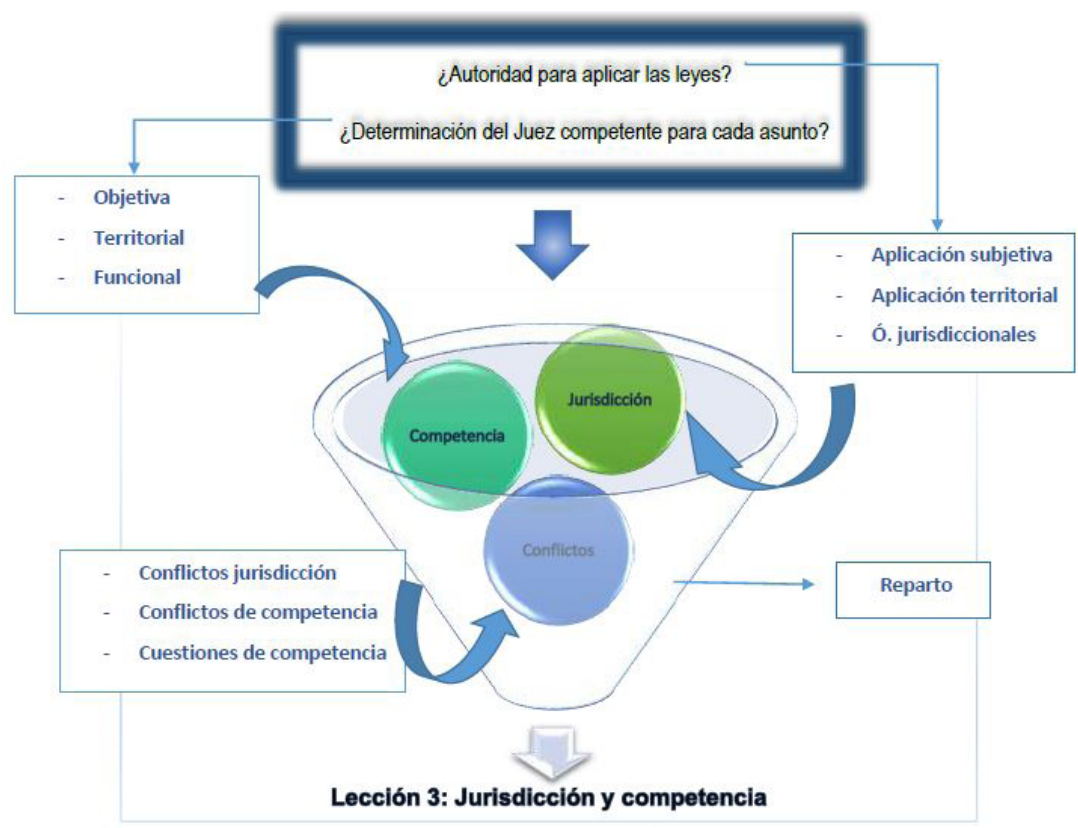

Figura 1. Mapa de contenidos

Ciclos de Mejora en el Aula (2020). Experiencias de Innovación Docente de la US Esta obra se distribuye con la licencia Creative Commons 


\section{Modelo metodológico posible}

No puede obviarse que el alumno posee algunos conocimientos dispersos sobre la primera parte del tema. Conoce las distintas ramas del derecho y, si bien no las delimita con perfección, puede acercarse al conocimiento por sí mismo. Esa es una de las ideas de este CIMA, por lo que el modelo metodológico se basa mucho en el encauzamiento de las ideas previas sobre este asunto que ya tiene el alumno, con el objetivo de que sea capaz de enfocarlas y aplicarlas en el futuro, es decir, aquí las ideas de los alumnos tienen un papel primordial. La disciplina, al poseer un fuerte carácter instrumental, requiere unas buenas bases organizadas de forma clara. No obstante, se ha intentado buscar un modelo metodológico que rompiera absolutamente con la estructura de clases que se venía impartiendo, para conseguir un máximo desarrollo del CIMA y ser capaz, a posteriori, de valorar el impacto de la introducción de formas alternativas de explicación de contenidos a alumnos muy habituados a una metodología poco cambiante. Como puede pensarse, no ha sido un reto sencillo, pues en clase ya se utilizaban medios audiovisuales e, incluso, plataformas como wooclap o kahoot. Junto a todas las cuestiones puramente técnicas, no puede olvidarse que nos encontramos formando a los jueces, fiscales, abogados, notarios o políticos del mañana, por lo que es fundamentar el inculcar el respeto a los valores y derechos fundamentales de un Estado de Derecho. Por todo ello, y como una forma de llevar el CIMA a su máximo rendimiento de forma que aúne todos los objetivos que se pretenden conseguir, se inserta un elemento externo que, a pesar de que hace más difícil la organización de contenidos, permite al alumno recibir conocimientos prácticos aplicativos a lo que está estudiando que, como ya dije ut supra, es un contenido árido. Este elemento viene proporcionado por los "Talleres para el fomento de relaciones saludables. Prevención de la violencia de género", que ofrece la propia Universidad de Sevilla en este primer cuatrimestre del curso 2020/2021.

Ciclos de Mejora en el Aula (2020). Experiencias de Innovación Docente de la US Esta obra se distribuye con la licencia Creative Commons 
La integración del taller como elemento externo y base para el desarrollo y aplicación del CIMA no permite establecer un patrón de modelo metodológico. Cada sesión presenta su propio modelo metodológico, en función del objetivo perseguido. No obstante, podríamos indicar de forma simple que todas seguirán las siguientes pautas:

\section{Secuencia de actividades programadas}

Se plantean una serie de actividades de diverso tipo que vienen reflejadas en la tabla siguiente. Se detalla en ella el tipo de actividad de que se trata, la duración dentro del tiempo de clase (teniendo en cuenta que la docencia se reparte en cuatro horas semanales: dos horas los lunes, una hora los miércoles y otra, los jueves), la fase metodológica a la que pertenece y el objetivo. Hemos insertado este último elemento para conseguir una mejor comprensión de la coherencia y la interrelación de las actividades dentro de la secuencia.

Tabla 1. Secuencia de actividades del ciclo de mejora

\begin{tabular}{|c|c|l|c|}
\hline \multicolumn{2}{|c|}{ SESIÓN 1 (2 horas) } & Objetivo \\
\hline Duración & $\begin{array}{c}\text { Fase } \\
\text { metodológica }\end{array}$ & \multicolumn{1}{|c|}{ Tipo de actividad } & Explicación CIMA \\
\hline $\mathbf{5}^{\mathbf{1 5}}$ & Introducción & $\begin{array}{l}\text { Exposición funcionamiento CIMA. } \\
\text { Los alumnos solo tienen que } \\
\text { escuchar con atención }\end{array}$ & $\begin{array}{c}\text { Obtener ideas } \\
\text { previas del } \\
\text { alumnado }\end{array}$ \\
\hline
\end{tabular}

Ciclos de Mejora en el Aula (2020). Experiencias de Innovación Docente de la US Esta obra se distribuye con la licencia Creative Commons 


\begin{tabular}{|c|c|c|c|}
\hline $40^{\prime}$ & $\begin{array}{l}\text { Actividad de } \\
\text { contraste }\end{array}$ & $\begin{array}{l}\text { Debate sobre: Europa Press, 2020, } \\
\text { Archivado el caso del accidente } \\
\text { mortal del ascensor del Hospital } \\
\text { de Valme de Sevilla, elDiario. } \\
\text { es; recuperado de: https://www. } \\
\text { eldiario.es/andalucia/sobreseida- } \\
\text { accidente-hospital-valme- } \\
\text { pedirlo_1_6042132.html }\end{array}$ & $\begin{array}{l}\text { Encontrar el } \\
\text { fundamento } \\
\text { a la división } \\
\text { de órdenes } \\
\text { jurisdiccionales } \\
\text { en razón a la } \\
\text { materia. }\end{array}$ \\
\hline $15^{\prime}$ & $\begin{array}{l}\text { Actividad de } \\
\text { contraste }\end{array}$ & $\begin{array}{l}\text { Lectura de textos (Gascón } \\
\text { Inchausti, 2019), sobre las materias } \\
\text { de: jurisdicción, reparto y órdenes } \\
\text { jurisdiccionales }\end{array}$ & $\begin{array}{l}\text { Situar los } \\
\text { conceptos de la } \\
\text { actividad anterior }\end{array}$ \\
\hline $30^{\prime}$ & $\begin{array}{l}\text { Actividad de } \\
\text { contraste }\end{array}$ & $\begin{array}{l}\text { Comentario de los textos, } \\
\text { exposición de las ideas principales }\end{array}$ & $\begin{array}{l}\text { Fijación de } \\
\text { conceptos }\end{array}$ \\
\hline $15^{\prime}$ & $\begin{array}{l}\text { Actividad de } \\
\text { sintesis }\end{array}$ & $\begin{array}{l}\text { Ejercicio de síntesis de todo lo } \\
\text { visto en la primera sesión. }\end{array}$ & Sintesis \\
\hline \multicolumn{4}{|c|}{ SESIÓN 2 y SESIÓN 3 (1 hora + 1 hora ) } \\
\hline Duración & $\begin{array}{c}\text { Fase } \\
\text { metodológica }\end{array}$ & Tipo de actividad & Objetivo \\
\hline $60^{\prime}$ & & & Concienciación \\
\hline $60^{\prime}$ & $\begin{array}{l}\text { Actividad de } \\
\text { contraste }\end{array}$ & $\begin{array}{l}\text { Taller de fomento de las relaciones } \\
\text { saludables y prevención de la } \\
\text { violencia de género }\end{array}$ & $\begin{array}{l}\text { del alumno } \\
\text { y extracción } \\
\text { de temas } \\
\text { relacionados con } \\
\text { la asignatura }\end{array}$ \\
\hline \multicolumn{4}{|c|}{ SESIÓN 4 (2 horas ) } \\
\hline Duración & $\begin{array}{c}\text { Fase } \\
\text { metodológica }\end{array}$ & Tipo de actividad & Objetivo \\
\hline $5^{\prime}$ & $\begin{array}{l}\text { Introducción/ } \\
\text { Ideas de los } \\
\text { alumnos }\end{array}$ & Comentario & $\begin{array}{l}\text { Reflexiones sobre } \\
\text { el taller }\end{array}$ \\
\hline 20' & $\begin{array}{l}\text { Actividad de } \\
\text { contraste }\end{array}$ & $\begin{array}{l}\text { Explicación del trabajo que } \\
\text { tendrán que realizar en grupos }\end{array}$ & $\begin{array}{c}\text { Comprensión del } \\
\text { trabajo }\end{array}$ \\
\hline $10^{\prime}$ & $\begin{array}{l}\text { Actividad de } \\
\text { contraste }\end{array}$ & $\begin{array}{l}\text { Organización de los grupos de } \\
\text { trabajo }\end{array}$ & - \\
\hline $85^{\prime}$ & $\begin{array}{l}\text { Actividad de } \\
\text { contraste }\end{array}$ & $\begin{array}{l}\text { Funcionamiento y trabajo de los } \\
\text { grupos }\end{array}$ & $\begin{array}{l}\text { Fomento del } \\
\text { trabajo grupal }\end{array}$ \\
\hline
\end{tabular}

Ciclos de Mejora en el Aula (2020). Experiencias de Innovación Docente de la US Esta obra se distribuye con la licencia Creative Commons Reconocimiento-NoComercial-SinObraDerivada $\quad 4.0$ Internacional (CC BY-NC-ND 4.0.) 


\begin{tabular}{|c|c|c|c|}
\hline Duración & $\begin{array}{c}\text { Fase } \\
\text { metodológica }\end{array}$ & Tipo de actividad & Objetivo \\
\hline 70' & $\begin{array}{l}\text { Actividad de } \\
\text { contraste }\end{array}$ & $\begin{array}{l}\text { Exposición de los distintos } \\
\text { trabajos. Votación (a través de la } \\
\text { herramienta sondeo de BBoard) } \\
\text { por los compañeros para la } \\
\text { elección de los tres trabajos } \\
\text { mejores, que tendrán puntuación } \\
\text { adicional al final de la asignatura }\end{array}$ & $\begin{array}{l}\text { Fomento de } \\
\text { la exposición } \\
\text { pública y el } \\
\text { hablar en público. } \\
\text { Ejercicios de } \\
\text { oratoria }\end{array}$ \\
\hline $15^{\prime}$ & $\begin{array}{l}\text { Hipótesis de } \\
\text { los alumnos/ } \\
\text { Teórica }\end{array}$ & $\begin{array}{l}\text { Explicación de los conflictos y } \\
\text { cuestiones de competencia }\end{array}$ & $\begin{array}{l}\text { Comprensión } \\
\text { de conceptos } \\
\text { relacionados }\end{array}$ \\
\hline 20' & $\begin{array}{l}\text { Actividad de } \\
\text { sintesis }\end{array}$ & $\begin{array}{l}\text { Ejercicio de síntesis de todo el } \\
\text { Tema } 3 \text { a través de uno de los } \\
\text { test contenidos en la web de } \\
\text { la Ludoteca jurídica (https:// } \\
\text { ludotecajuridica.es/) }\end{array}$ & $\begin{array}{l}\text { Fijación de } \\
\text { conceptos y } \\
\text { sintesis }\end{array}$ \\
\hline $15^{\prime}$ & $\begin{array}{l}\text { Ideas finales de } \\
\text { los alumnos }\end{array}$ & Herramienta de diagnóstico & $\begin{array}{c}\text { Comparación } \\
\text { ideas iniciales/ } \\
\text { finales. Evaluación } \\
\text { del aprendizaje }\end{array}$ \\
\hline
\end{tabular}

Cuestionarios inicial-final:

herramienta de diagnóstico

La herramienta de diagnóstico que voy a utilizar está conformada por las preguntas siguientes (la pregunta 6 solo se introduce en el cuestionario final, con el objetivo de que los alumnos valoren la aplicación y el desarrollo del CIMA):

1. ¿Qué es para ti la jurisdicción?

2. ¿A quién crees que se aplica?

3. ¿Existen órdenes jurisdiccionales? En el caso de que creas que existen: ¿cuáles son? y ¿cuál es su objetivo?

Ciclos de Mejora en el Aula (2020). Experiencias de Innovación Docente de la US Esta obra se distribuye con la licencia Creative Commons 
4. ¿Pueden existir conflictos en la atribución y conocimiento de los asuntos en el poder judicial? ¿Podrías citar algún ejemplo?

5. ¿Por qué te puede corresponder un juez u otro?

6. Si tú fueras el profesor de esta asignatura... ¿Qué mejorarías o cambiarías para la explicación de este Tema 3?

\section{Aplicación del Ciclo de Mejora en el Aula}

\section{Resumen de las sesiones}

Las primeras cuatro sesiones se siguieron conforme a los tiempos y cronología establecidos en la tabla de diseño del CIMA.

En la primera sesión los alumnos se muestran muy interesados en el nuevo sistema de la clase, basado en una participación más activa por su parte, aunque nos encontramos todos con la dificultad de la clase bimodal. Consideran que el cuestionario tiene una dificultad media. Les gusta mucho acercarse a un caso real como es el caso del ascensor del Hospital de Valme, por lo que se enciende mucho el debate y se muestran muy participativos. Se crea un ambiente de trabajo que los hace pensar y eso se muestra en la atención que prestan a la parte relativa al análisis de los textos que hacemos a continuación. De ellos, sacan las ideas más importantes que van integrando con las ideas preconcebidas que poseían. Empiezan a distinguir la jurisdicción ordinaria de las especiales. Para terminar, se realiza un ejercicio de síntesis donde repasamos todos los conceptos. Me indican que es el día que más se han enterado de todos los contenidos de lo que llevamos de curso y que con este sistema las clases son más amenas y entretenidas.

Ciclos de Mejora en el Aula (2020). Experiencias de Innovación Docente de la US Esta obra se distribuye con la licencia Creative Commons 
La segunda y tercera sesión están dedicadas al taller de fomento de las relaciones saludables y prevención de violencia de género en los jóvenes universitarios. Esta actividad alcanza un alto grado de inscripción (los alumnos tienen que inscribirse individualmente), puesto que no están acostumbrados a la integración de un elemento externo en las clases. Además, la profesora encargada del taller proviene de la Facultad de Enfermería, lo que produce desconcierto en el alumnado. La pregunta que subyace es la siguiente: ¿cómo vamos a relacionar este taller con el derecho procesal y la materia a estudiar en este tema?

A pesar de que el contenido no es de carácter jurídico, los alumnos extraen los temas más importantes con contenido y relevancia judicial: sexting, ciberacoso, ciberviolencia, muerte materna por problemas colaterales, repercusión de la violencia de género en hijos e hijas...Los alumnos se muestran muy interesados. Además, el hecho de recibir un certificado de la actividad (externo a las clases y previa inscripción) les produce una motivación extra. De todas formas, al recoger las impresiones me doy cuenta de que están muy concienciados y me llama la atención que hablen y comenten más los chicos que las chicas. con este taller se busca fomentar también los contenidos actitudinales.

La cuarta sesión comienza con la elección de los temas y la formación de los grupos. Además, se explica el trabajo. Tienen problemas para formar los grupos, pues aún estamos en formato bimodal y muchos ni siquiera se conocen. Por ello, tomo la decisión de que sea un trabajo de carácter voluntario con grupos más o menos amplios y a su elección. Surgen 9 grupos. Las reticencias empiezan cuando entienden el trabajo que hay que hacer, porque ellos son conscientes (al igual que yo) que es un trabajo difícil. Sin embargo, la motivación de conseguir puntos extra los anima mucho a participar y deciden ponerse a trabajar de inmediato. 
Los problemas surgen a partir de la sesión 5 (dejo una semana por medio donde sigo avanzando en el temario con clases fuera del CIMA, ya que necesitan tiempo para realizarlo). Debido a la implementación de las medidas establecidas por la Orden de 8 de noviembre de 2020, por la que se modulan los niveles de alerta 3 y 4 como consecuencia de la situación crítica epidemiológica derivada del COVID-19 en la Comunidad Autónoma de Andalucía, ya pasamos al modelo de clase online, por lo que todo se complica. Nunca han expuesto trabajos de forma virtual. A pesar de ello, me doy cuenta de que los trabajos tienen un nivel muy alto y que solo da tiempo a 2 o 3 grupos por hora. Lo que yo pensaba que se podía hacer en hora y media, pasa a ser seis horas. Eso ha sido lo más complicado de gestionar, puesto que la exposición me ha llevado más tiempo del esperado. Sin embargo, no quise poner un límite de tiempo porque, como ya he expuesto, la calidad de los trabajos ha sido altísima, tanto que han avanzado en el temario, saliéndose de los contornos del tema que estábamos desarrollando en el CIMA y entrando de lleno en los contenidos de la disciplina procesal, por lo que he preferido que expusieran con libertad. Observo problemas de tiempo y cronología, pero no de calidad en la aprehensión de los contenidos. Al revés.

Las votaciones sobre la elección del mejor trabajo se llevan a cabo muy bien y al final exponen 8 grupos (aunque originariamente surgieron 9 , uno de los grupos se retira). Había indicado que solo 3 grupos podrían tener puntos extra, pero todos los trabajos son buenos, por lo que reparto mucho más la puntuación. Se muestran muy contentos y expresan lo que se estaba viendo: habian aprendido mucho más y les había empezado a interesar la asignatura. No obstante, hay un reducido grupo que muestra su disconformidad por la exigencia del trabajo.

Ciclos de Mejora en el Aula (2020). Experiencias de Innovación Docente de la US Esta obra se distribuye con la licencia Creative Commons 
En la última sesión, explico los conflictos y cuestiones de competencia, pero ya sin dificultad, pues lo entienden muy bien por la realización del trabajo. También les gusta mucho el cuestionario que plantea la web de ludoteca jurídica, pues pueden participar de forma colectiva y les sirve para fijar los conocimientos. Lo utilizamos como ejercicio de sintesis. Realizan el cuestionario final y me exponen su satisfacción con este nuevo sistema de clases, puesto que la gran mayoría considera que aprende más que con el método tradicional y que se han metido de lleno en el tema y la materia.

Se han podido realizar todas las actividades previstas. Sin embargo, lo que originariamente era un CIMA de ocho horas ha pasado a ser un CIMA de doce horas.

\section{Evaluación del aprendizaje de los alumnos}

ANÁLISIS: participan 48 alumnos en el inicial y 35 en el final. Es un cuestionario totalmente anónimo. Los alumnos muestran reticencias, puesto que nunca han realizado algo así. La evaluación de los cuestionarios se ha realizado siguiendo el modelo previsto por Rivero y Porlán (2017). Los resultados obtenidos se muestran en las figuras siguientes (por su interrelación las preguntas 1 y 2 se valoran conjuntamente, al igual que la 4 y la 5):

\section{Preguntas 1 y 2: Jurisdicción \\ Herramienta de diagnóstico inicial}

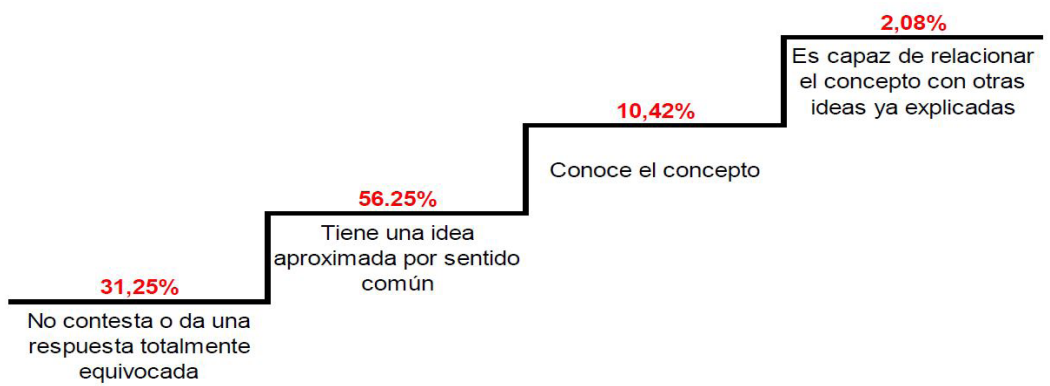

Figura 3. Escalera de aprendizaje inicial (preguntas 1 y 2)

Ciclos de Mejora en el Aula (2020). Experiencias de Innovación Docente de la US Esta obra se distribuye con la licencia Creative Commons 
$28,57 \%$

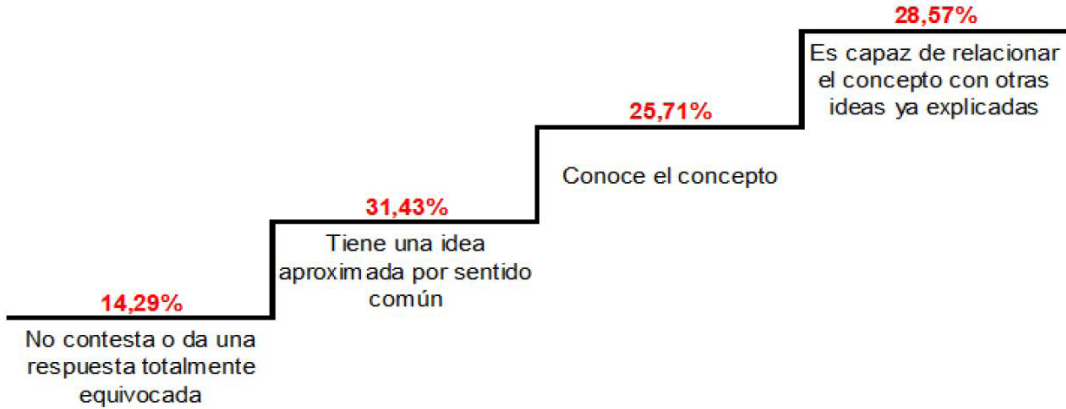

Figura 4. Escalera de aprendizaje final (preguntas 1 y 2 )

\section{Pregunta 3: Órdenes jurisdiccionales \\ Herramienta de diagnóstico inicial}

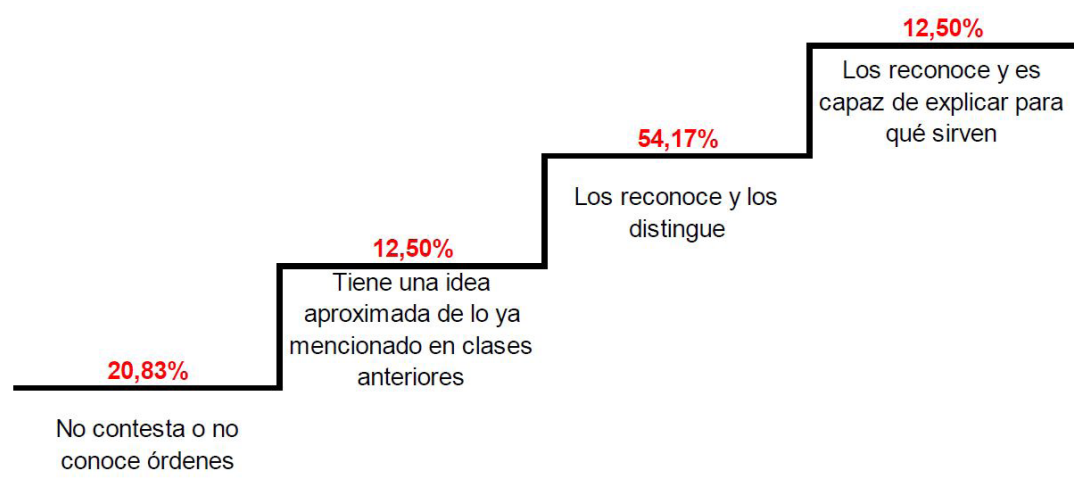

Figura 5. Escalera de aprendizaje inicial (pregunta 3)

\section{Herramienta de diagnóstico final}

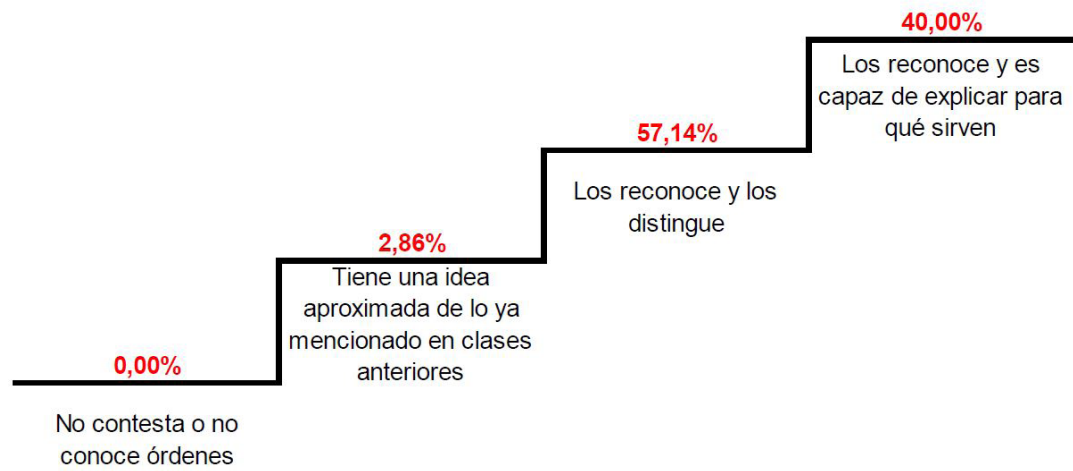

Figura 6. Escalera de aprendizaje final (pregunta 3)

Ciclos de Mejora en el Aula (2020). Experiencias de Innovación Docente de la US (C) Esta obra se distribuye con la licencia Creative Commons 


\section{Preguntas 4y 5:Competencias y conflictosjurisdicción/ competencia}

\section{Herramienta de diagnóstico inicial}

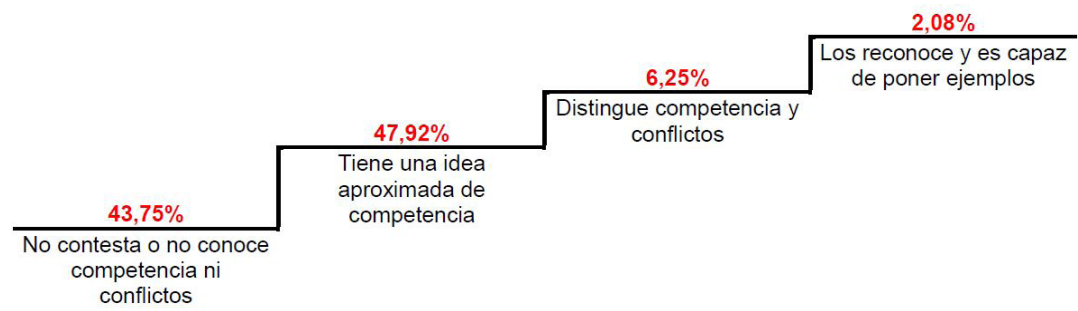

Figura 7. Escalera de aprendizaje inicial (preguntas 4 y 5)

\section{Herramienta de diagnóstico final}

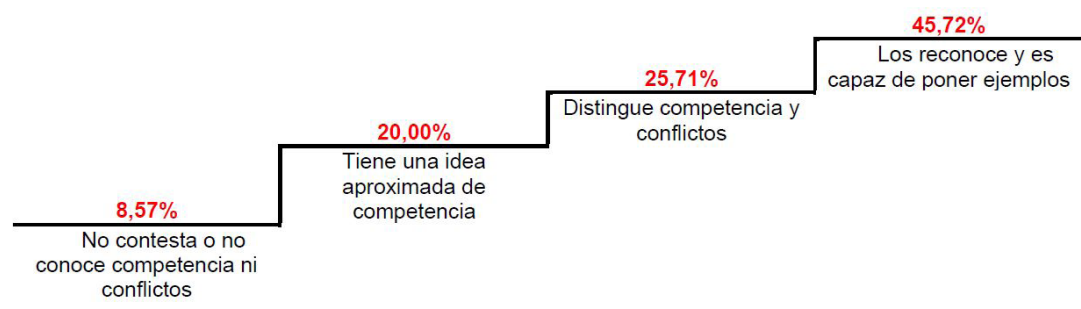

Figura 8. Escalera de aprendizaje final (preguntas 4 y 5)

\section{Pregunta 6: Valoración del CIMA por el alumnado (Solo cuestionario final)}

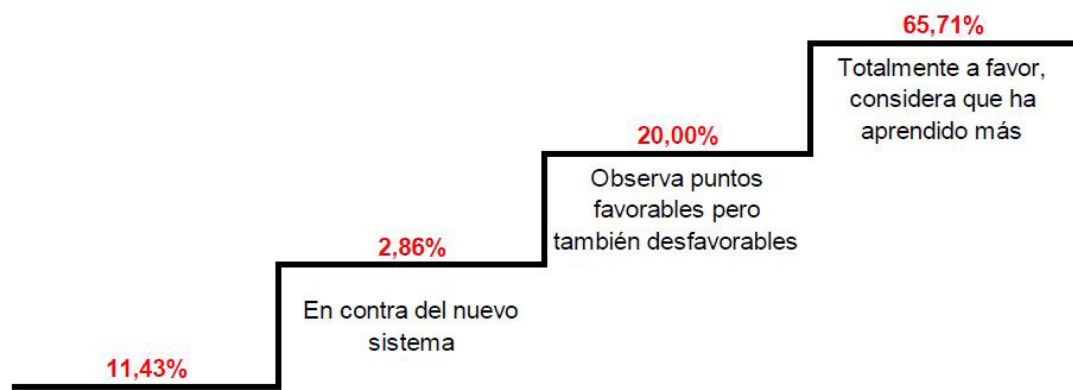

No contesta

Figura 9. Valoración (pregunta 6)

En relación a las dos primeras preguntas se observa que el desconocimiento era generalizado. La mayoría

Ciclos de Mejora en el Aula (2020). Experiencias de Innovación Docente de la US (c) Esta obra se distribuye con la licencia Creative Commons 
desconocía o solo tenía una ligera idea de lo que significaba el término "jurisdicción". Con la aplicación del CIMA, más del 50\% del alumnado es capaz de definir el concepto y el $28,57 \%$ incluso puede poner ejemplos (hay que tener en cuenta que era el tema más complicado de este CIMA).

En lo referente a la pregunta 3, el cuestionario final nos muestra que más del $97 \%$ de los alumnos conoce ya los órdenes jurisdiccionales y ninguno está en el escalón más bajo. Si bien es cierto que este tema había surgido (aunque de forma transversal) en lecciones anteriores, en el cuestionario inicial más del 30\% del alumnado era incapaz de fijar el concepto. En este caso los resultados son muy satisfactorios, ya que se trata de una de las cuestiones más importantes de toda la asignatura. Su comprensión e interiorización es fundamental para los contenidos que vendrán después (tanto en este curso como en los superiores).

Las preguntas 4 y 5 son las que muestran una mejor evolución del alumnado. Esta evolución puede derivarse de la elaboración del trabajo que han realizado, puesto que las preguntas constituyen los elementos fundamentales del mismo. De un $2 \%$ inicial que era el único capaz de reconocer la competencia y los conflictos y poner ejemplos, en el cuestionario final este grupo aumenta más de un $43 \%$. En mi opinión, éstas son las preguntas que mejor reflejan el impacto del CIMA en el alumno.

Por último, quiero hacer referencia a la pregunta 6 . Como ya se ha dicho, esta pregunta fue incluida en el cuestionario final para la valoración del CIMA. Los resultados reflejan el gran interés despertado, siendo muy irrelevante el porcentaje de alumnos que se muestra directamente en contra de su aplicación. Además, el 65\% del total de alumnos muestra un actitud muy positiva frente a este sistema metodológico (hay que tener en cuenta que durante la aplicación del CIMA se ha pasado del sistema bimodal al online).

Ciclos de Mejora en el Aula (2020). Experiencias de Innovación Docente de la US Esta obra se distribuye con la licencia Creative Commons 
En lo relativo a la evaluación material del alumnado, ésta se ha llevado a cabo a través de la valoración del trabajo grupal, realizándose una evaluación en la que han participado también los alumnos, votando los mejores trabajos en base a la exposición, el contenido, la claridad...

\section{Evaluación del Ciclo de Mejora en el Aula}

Puede afirmarse que la aplicación de este CIMA ha arrojado unos resultados muy positivos que, incluso podríamos clasificar de sorprendentes. Los alumnos han ido más allá en la asignatura, adelantándose y profundizando en temas que desconocían: han "buceado" por sí mismos en una disciplina complicada. Considero además que ha sido muy útil el integrar un elemento externo (taller). Con él se buscaba que los alumnos tomaran conciencia de la problemática y, por sus propias ideas, llegasen al orden jurisdiccional penal (que era uno de los objetivos clave, pues en el curso siguiente tendrán una materia dedicada únicamente al conocimiento de la materia procesal penal). Normalmente se les hace muy dificil integrar los conocimientos sustantivos con los procesales, por lo que estos talleres son idóneos para reflexionar sobre la materia y su proyección procesal. Desde la realidad cotidiana han llegado hasta el derecho procesal (cuestión que me manifestaban como imposible en un principio) y lo han proyectado en un trabajo grupal. Además, el recibir un docente externo capta su atención, los acerca a la realidad desde otro punto de vista y fomenta su motivación. Mientras reflexionan sobre aspectos de su vida privada, están obteniendo los conocimientos necesarios para superar la asignatura y, lo que es más importante, sentando las bases de conocimientos sobre los que tendrán que profundizar en un futuro próximo. Siempre que pueda recurriré a elementos externos que estén en conexión con la materia.

Ciclos de Mejora en el Aula (2020). Experiencias de Innovación Docente de la US Esta obra se distribuye con la licencia Creative Commons 
Así, como principios didácticos inspiradores y que han guiado esta experiencia docente debo destacar, por un lado, los principios de autonomía y socialización, en tanto que he querido que el alumno pueda encontrar el interés en la materia por sí mismo, relacionando conceptos ya conocidos por él y, a la vez, fomente sus capacidades para trabajar en grupo y, por otro, el principio de actividad, ya que ha sido el impulso del propio alumnado el que les ha permitido adentrarse y conocer la materia: su trabajo ha sido el motor de la docencia.

Si bien es una realidad que la experiencia ha sido muy satisfactoria, no debe obviarse el asunto del tiempo. Observo como aspecto fundamental a tener en cuenta que la aplicación generalizada de CIMAS durante el curso ha de conllevar una reducción de temario, por lo que me planteo la dicotomía temario completo-buena interiorización de conceptos por el alumnado. Es cierto que este dilema siempre se responde con la necesidad de una mejor comprensión de contenidos (y para ello entiendo que el CIMA es la mejor opción), pero eso puede producirles perjuicios en comparación con otros grupos de alumnos, por lo que creo que necesita una mayor reflexión por mi parte (adecuación de contenidos, más trabajo individual del alumno...). De todas formas, lo que la aplicación del CIMA me ha demostrado es que un alumno sólo necesita motivación y una pautas de sintesis con sentido y reflexión acerca de lo que se está explicando para alcanzar conocimientos que pensamos que están muy alejados de su nivel. No pongamos listones de nivel a nuestros alumnos, dejémosles avanzar por sí mismos: los resultados sorprenden.

Palabras clave: Derecho Procesal I, Grado en Derecho, docencia universitaria, experimentación docente

Key words: Procedural Law I, Law Degree, university teaching, university teaching experimentation

Ciclos de Mejora en el Aula (2020). Experiencias de Innovación Docente de la US Esta obra se distribuye con la licencia Creative Commons 


\section{Referencias bibliográficas}

Bain, K. (2007). Lo que hacen los mejores profesores universitarios. Valencia: Publicaciones Universidad de Valencia.

Finkel, D. (2008). Dar clase con la boca cerrada, Valencia: Publicaciones Universidad de Valencia.

Gascón Inchausti, F. (2020). Derecho Procesal Civil: Materiales para el estudio. Edición digital, copyleft

Gimeno Sendra, V., Díaz Martínez, M. y Calaza López, S. (2020). Introducción al Derecho Procesal. Valencia: Tirant Lo Blanch.

Martín Ostos, J. (2020). Introducción al Derecho Procesal. Sevilla: Astigi.

Porlán R. (Coord.). (2017). Enseñanza Universitaria. Cómo mejorarla. Madrid: Ediciones Morata.

Rivero, A. y Porlán, R. (2017). La evaluación de la enseñanza universitaria. En R. Porlán (Coord.), Enseñanza Universitaria. Cómo mejorarla (pp. 73-92). Madrid: Ediciones Morata.

Ciclos de Mejora en el Aula (2020). Experiencias de Innovación Docente de la US Esta obra se distribuye con la licencia Creative Commons 